\title{
A multidimensional scaling program for distinctiveness and similarity based on stimulus classification and information measurement
}

\author{
D. C. DONDERI \\ McGill University, Montreal, Quebec, Canada
}

\begin{abstract}
When observers classify a set of multidimensional items on the basis of similarity, they generate information measures that are isomorphic to item vectors whose lengths correspond to the judged distinctiveness of each item and whose angles define the position of each item in a euclidean similarity space. The PROSCALE computer program calculates the item vectors and spatial positions from similarity classification data and then generates and rotates orthogonal dimensions of the similarity space. PROSCALE can also use variables associated with each item to generate oblique dimensions that span the space. PROSCALE carries out multidimensional similarity scaling or unidimensional magnitude estimation scaling on as many as 70 items for as many as 50 observers.
\end{abstract}

Multidimensional scaling (MDS) is a statistical technique for describing some of the psychological characteristics of a set of items (which can be words, objects, or pictures) by generating a multidimensional similarity space that contains the items. Euclidean space is the most common framework, although non-euclidean spaces have also been used (Shepard, 1964). Euclidean space makes it easy to visualize the attributes that differentiate the items. The attributes are dimensions that establish a coordinate system for the space. The point that represents an item in the space is projected onto each dimension to the extent that the item possesses the attribute represented by that dimension. Interitem distances are calculated from the projection of each item on each dimension, and item similarity is the complement or reciprocal of interitem distances in the space.

Conventional MDS requires that observers compare every pair of items in order to establish interitem distances. Then the item pair distances are analyzed to establish the dimensionality and structure of the space. For $n$ items, $\left(n^{2}-n\right) / 2$ comparisons are required, so the observer's task becomes quadratically longer as the number of items increases. As a result, conventional MDS is usually limited to item sets of modest size (5-10 items).

Most of the program described here was developed while the author was on sabbatical leave at the Pulp and Paper Research Institute of Canada (PPRIC), Pointe Claire, Quebec, with which the author is affiliated as a Faculty Associate. Software costs to develop the program were underwritten by PPRIC. The author thanks Joseph A. Aspler, Head of the Printing Research Group, PPRIC, for his hospitality at PPRIC and for his interest in this work. The author also thanks Yoshio Takane and J. O. Ramsay of the McGill University Department of Psychology for their interest and for their help. Correspondence should be addressed to D. C. Donderi, Department of Psychology, McGill University, 1205 Dr. Penfield Ave., Montreal, PQ, Canada H3A lBI (e-mail: donderi@hebb.psych.mcgill.ca).
Another method of generating a euclidean space from similarity classifications was described by Donderi (1988). The observer divides a set of simultaneously displayed items into smaller subsets or groups, so that "the pictures in each group are alike but each group is different from the other groups in some way that is clear to you" (p. 580). Either one classification from multiple observers or multiple classifications from one observer are then analyzed using information theory to generate a distinctiveness measure for each item and a distance measure for each pair of items.

The judgment task time for similarity classification does not increase quadratically with the number of items. Experience shows that observers can classify as many as 45 simultaneously presented items within $30 \mathrm{~min}$ (Donderi \& Aspler, 1994) and as many as 70 in about $1 \mathrm{~h}$. Range and adaptation effects are ubiquitous in scaling and preference judgments (Helson, 1947; Mellers \& Cooke, 1996; Stevens, 1975, pp. 268-296). One way to neutralize range and adaptation effects is to present as many of the relevant items as possible and, for visual items, to keep as many simultaneously visible as possible (Cleveland, Harris, \& McGill, 1983). If a large range of relevant items is judged simultaneously, then there are fewer other items that, had they been present, might have changed the results. The similarity classification technique takes less time per item than conventional MDS and so makes it easier to present a large range of items.

Donderi (1988) demonstrated empirical agreement between a conventional MDS similarity space and a space based on the similarity classification of 10 color photographs. Interitem distances derived from all-pairs MDS comparisons correlated highly with the interitem distances derived from similarity classification, and direct judgments of distinctiveness obtained as a supplement to the all-pairs MDS task correlated highly with measures 
of distinctiveness derived from the similarity classification task.

Although the 1988 paper established the empirical equivalence of conventional and stimulus classification MDS, it did not introduce a new MDS program. Instead, similarity classification based on information measurement was used to generate interstimulus distances among all item pairs and between each item and the "origin" of the space. Then, an existing metric MDS program (Multiscale; Ramsay, 1991) was used to analyze the complete set of interstimulus distances and to extract the appropriate number of dimensions. ${ }^{1}$ More recent similarity classification applications (Donderi \& Aspler, 1994) also used Multiscale to analyze the results.

This paper introduces a new computer program that carries out an MDS analysis of similarity classification data for as many as 70 items and as many as 50 observers. The program takes advantage of the fact that similarity classification analysis establishes a unique common origin, and unique relative orientations, for the vectors that describe the items in the space. Given the length and relative orientations of the item vectors in the space, conventional factor analytical methods can then be used to find, evaluate, and rotate the principal component dimensions spanning the space. This novel property of similarity classification MDS, which is found in only one earlier timeconsuming MDS technique (Ekman, Engen, Künnapas, $\&$ Lindman, 1963), gives the new program the advantage of defining both distinctiveness and similarity as outcomes of the same simple task.

The first section of the present paper reviews previous developments in similarity measurement and MDS that are relevant to this work. Asymmetrical similarity measures are given particular attention. Then the transformation from similarity classification to euclidean space is described, and the computer program that carries out the transformations and produces the scaling results is outlined. Finally, theoretical and practical aspects of the new technique are discussed, and some applications are described.

\section{SOME MEASURES OF SIMILARITY}

\section{Pair-Comparison Similarity Measures}

MDS spaces are usually defined by measurements on pairs of items. Observers are asked to judge the distance between each item pair. After various transformations, the ensemble of interpair distances is used to estimate the dimensionality of the space (e.g., Ramsay, 1991).

There are disadvantages in constructing a euclidean space from item pair distance measures. Because it takes $\left(n^{2}-n\right) / 2$ measurements to unambiguously define the symmetrical distance pairs among $n$ items, a set of 40 items requires 780 comparisons. Although many pairs in a sparse data matrix can be estimated rather than measured (Spence \& Domoney, 1974) MDS is not often used with large item sets.

Another disadvantage is that when a euclidean similarity space is defined only by item pair distances, the inter- item distances define neither an origin for the space nor a unique vector from the origin corresponding to the position of each item.

\section{Set-Theoretical Similarity Models}

Tversky (1977) recognized the value of spatial models for measuring the similarity of items composed of what Garner (1974) called integral dimensions: dimensions such as hue, saturation, and brightness that combine to give a unitary sensory or conceptual experience of the stimulus. However, Tversky criticized spatial MDS models for their insensitivity to asymmetries in perceptual or cognitive characteristics that are elicited when two items are compared. He developed an alternative settheoretical similarity model for stimuli composed of a small number of separable dimensions that could be easily analyzed out of the stimulus, such as the mouth, nose, eyes, and eyebrows of a simplified schematic face. Tversky's theory emphasizes the total size of the feature set that characterizes each stimulus, as well as the overlap between the feature sets of different stimuli. His very general formulation of the similarity between two stimuli, expressed in words, is that similarity is a weighted function of the set of features that two stimuli have in common minus the weighted functions of the sets of features that each stimulus has independently of the other.

Tversky correctly claimed that one of the main advantages of his approach is that it accounts for asymmetries of judgment between stimuli of different complexity (number of attributes). Cuba was judged to be similar to the Soviet Union, but not vice versa, because Cuba had very few attributes independent of those it shared with the Soviet Union, whereas the Soviet Union had many attributes independent of those it shared with Cuba. Tversky and Gati (1982) challenged the applicability of euclidean spatial distance models to separable dimensions by demonstrating that "the addition of the same feature to a pair of perceptual or conceptual stimuli increases the similarity between them" (p. 153). They also demonstrated the failure of judgments made to stimulus sets constructed from separable dimensions to meet the combined requirements of the triangle inequality and unidimensional additivity.

\section{MDS With Asymmetrical Similarities}

Ekman et al. (1963) had already developed an MDS model that answered some of the criticisms made by Tversky. Their experimental task required observers to "estimate, on a suitable scale, which proportion of $j$ is contained in $i$ (e.g., 'sixty-five percent of that red is present in this orange') and vice versa" (p. 3). These ratio measurements were then used to calculate the projection of a red stimulus vector on an orange vector, and vice versa.

The Ekman et al. model calculates the cosines of the angles between every pair of stimulus vectors using the complete set of asymmetrical ratio measurements. The result is a measure of stimulus similarity that depends both on the "quality" of the difference between two stimuli (the cosine of the angle between the stimulus vectors) 
and the "quantity" of each stimulus (the length of the vector). Since Ekman et al.'s quality similarity measures are based on the angles of vectors relative to each other in space, factor analysis can be used to derive a set of rotated orthogonal axes that span the similarity space.

The Ekman et al. method requires two measurements for each stimulus pair and demands much time and effort from the observer (e.g., a set of 40 items requires 1,560 judgments). However, it does completely describe the stimuli as vectors in a euclidean space with a natural origin, and it generates both symmetric and asymmetric measures of stimulus similarity.

\section{SIMILARITY CLASSIFICATION AND EUCLIDEAN DISTANCE}

A euclidean space is also generated by classifying a set of items on the basis of similarity. The set is partitioned by the instruction, "Place the items into groups, so that each group has something in common which distinguishes it from all of the other groups" (Donderi, 1995b; Donderi \& Aspler, 1994). Measures on the partitioned set generate a space with three important properties: (1) there is an origin that is the zero point of both the item vectors and the dimension vectors that span the space, (2) the distinctiveness of each item is the length of the vector from the origin to the point representing the item, and (3) the distance between every pair of items is the distance between the points representing the items in the space. Like the Ekman et al. method, the similarity classification method measures both quantity (distinctiveness) and quality (vector angle cosines) in a euclidean space with a defined origin.

\section{From Similarity to Information to Distance}

Similarity classifications are transformed into distances by calculating the information in each item on the basis of its classification. The definition of information is the reduction of uncertainty due to classification. ${ }^{2}$ This is best understood by an example. The uncertainty of a set of eight items is $\log _{2} 8=3$ bits. Suppose an observer groups two of the eight items, A and B, together on the basis of similarity. Items A and B are now members of a smaller subset of two items. The uncertainty of that subset is $\log _{2} 2=1$ bit. The information gained by classifying Items $\mathrm{A}$ and $\mathrm{B}$ together is equivalent to the uncertainty reduced because they are now members of a smaller subset. This quantity is always calculated relative to the uncertainty of the original set. It is simply the difference between the uncertainty of the original set and the uncertainty of the similarity subset. In this case, the information gained is 3 bits -1 bit $=2$ bits. Symbolically, $H(\mathrm{~A})=H(\mathrm{~B})=2$, where the $H$ is the conventional symbol for information, and the letter in parentheses identifies the item. As explained above, the definition of $H(\mathrm{~A})$ used in this paper is $H(\mathrm{~A})=\log _{2} N(\mathrm{~S})-\log _{2} N\left(\mathrm{G}_{\mathrm{A}}\right)$, where $N(\mathrm{~S})$ is the number of items in the original set and
$N\left(\mathrm{G}_{\mathrm{A}}\right)$ is the number of items in the similarity group of which $\mathrm{A}$ is a member.

The information theory symbol for the information in Item $B$ that is independent of the information in Item A is $H \mathrm{a}(\mathrm{B})$, where the capital letter in parentheses indicates the item under consideration, and the small letter indicates the item against which the independent information is measured. We define information in $A$ to be independent of $B$ (and vice versa) if $A$ and $B$ are classified into different groups. In other words, if $\mathrm{A}$ and $\mathrm{B}$ are in different groups, $H \mathrm{~b}(\mathrm{~A})=H(\mathrm{~A})$, and $H \mathrm{a}(\mathrm{B})=H(\mathrm{~B})$. The term $H \mathrm{~b}(\mathrm{~A})+H \mathrm{a}(\mathrm{B})$ defines the sum of the information in Items $A$ and $B$ that is independent of information in the other item. If $\mathrm{A}$ and $\mathrm{B}$ are in different groups, $H \mathrm{~b}(\mathrm{~A})+H \mathrm{a}(\mathrm{B})=$ $H(\mathrm{~A})+H(\mathrm{~B})$. We define information in $\mathrm{B}$ to be dependent on $A$ (and vice versa) if $A$ and $B$ are classified in the same group. In this case, $H \mathrm{a}(\mathrm{B})=0$, and $H \mathrm{~b}(\mathrm{~A})=0$.

The total information in a pair of items is symbolized as $H(\mathrm{~A}, \mathrm{~B})$. Information theory establishes that $H(\mathrm{~A}, \mathrm{~B})=$ $H(\mathrm{~A})+H \mathrm{a}(\mathrm{B})=H(\mathrm{~B})+H \mathrm{~b}(\mathrm{~A})$ (see Attneave, 1959 , pp. 49-50). The total information in a pair of items equals all the information in one item plus all the information in the second item that is independent of the information in the first.

Thus, when $\mathrm{A}$ and $\mathrm{B}$ are classified separately, $H \mathrm{~b}(\mathrm{~A})=$ $H(\mathrm{~A})$, and $H \mathrm{a}(\mathrm{B})=H(\mathrm{~B})$. Then, $H(\mathrm{~A}, \mathrm{~B})=H(\mathrm{~A})+$ $H \mathrm{a}(\mathrm{B})=H(\mathrm{~B})+H \mathrm{~b}(\mathrm{~A})=H(\mathrm{~A})+H(\mathrm{~B})$. When $\mathrm{A}$ and $\mathrm{B}$ are classified together, $H \mathrm{a}(\mathrm{B})=H \mathrm{~b}(\mathrm{~A})=0$, and $H(\mathrm{~A}, \mathrm{~B})$ $=H(\mathrm{~A})+H \mathrm{a}(\mathrm{B})=H(\mathrm{~A})+0=H(\mathrm{~A})$, and $H(\mathrm{~A}, \mathrm{~B})=$ $H(\mathrm{~B})+H \mathrm{~b}(\mathrm{~A})=H(\mathrm{~B})+0=H(\mathrm{~B})$; therefore, $H(\mathrm{~A}, \mathrm{~B})=$ $H(\mathrm{~A})=H(\mathrm{~B})$

Claude Shannon suggested that the information measure $H \mathrm{a}(\mathrm{B})+H \mathrm{~b}(\mathrm{~A})$ be used as a measure of the distance between Items A and B (Parke \& Sampson, 1951). Shannon's measure is the sum of the information in each item that is independent of the information in the other. Using our definitions, Shannon's measure equals zero when A and $\mathrm{B}$ are classified together, and it equals $H(\mathrm{~A})+H(\mathrm{~B})$ when A and B are classified separately.

In order to generate a complete isomorphism between information measures and euclidean distances, it was necessary to modify Shannon's distance measure (Donderi, 1988). I took the square root of the Shannon measure to equal the distance in similarity space between any pair of Items $A$ and $B$. In information measure terms, the distance between the two items, $d(\mathrm{~A}, \mathrm{~B}) \equiv[\mathrm{Ha}(\mathrm{B})+$ $H \mathrm{~b}(\mathrm{~A})]^{1 / 2}$. The distance from each item to the origin, $d(\mathrm{O}, \mathrm{A}) \equiv[H(\mathrm{~A})]^{1 / 2}$, and $d(\mathrm{O}, \mathrm{B}) \equiv[H(\mathrm{~B})]^{1 / 2}$, measures the distinctiveness of each item. That these measures define a consistent euclidean space was demonstrated in Donderi (1988).

A single similarity classification always produces an information-distance isomorphism in which all the items in each group occupy an identical position on one of the orthogonal dimensions of the space, which has as many dimensions as there are classification groups. The items in the smaller groups iie farther from the origin, and the 
items in the larger groups lie closer to the origin, on their respective orthogonal dimensions. When multiple similarity classifications are averaged, the individual item vector positions and orientations are averaged, and, as a result, both the lengths and the relative orientations of the resulting item vectors may vary widely from the grouping and orthogonality found after a single classification.

The information-distance isomorphism is illustrated in Figure 1. The point $O$ is the origin of the euclidean space. The vector $O A$ defines the average position of Item $A$, and the vector $O B$ defines the average position of Item B. The scalar distance OA measures the distinctiveness of Item $A$, and $O B$ measures the distinctiveness of Item $\mathrm{B}$. The distance $\mathrm{AB}$ measures the distance between Item Pair $A B$ in the similarity space. The isomorphism between information measures and distances is shown in Figure 1.

The cosine of the angle between Vectors A and B at the origin is defined by the cosine law:

$$
\cos \theta=\frac{d(\mathrm{~A}, \mathrm{~B})^{2}-d(\mathrm{O}, \mathrm{A})^{2}-d(\mathrm{O}, \mathrm{B})^{2}}{2[d(\mathrm{O}, \mathrm{A}) * d(\mathrm{O}, \mathrm{B})]} .
$$

Since all of the distances needed to calculate angle cosines have information measure equivalents, similarity classification generates angle cosines for every pair of vectors. The cosine of the vector angle $\mathrm{AOB}$ is equivalent in factor analysis to the correlation between Items $A$ and B (Gorsuch, 1983).

\section{Asymmetrical Similarity From Similarity Classification}

Similarity classification also generates asymmetrical measures of similarity. In the Ekman et al. (1963) complete euclidean space, the projection of Vector $A$ onto Vector B, or, in other words, the proportion of Stimulus A that is represented in Stimulus $\mathrm{B}$, equals $h_{\mathrm{A}} \cos \theta$, where $h$ is the length of Item Vector $A$, and $\theta$ is the angle between Vectors A and B. Because Vectors A and B are not

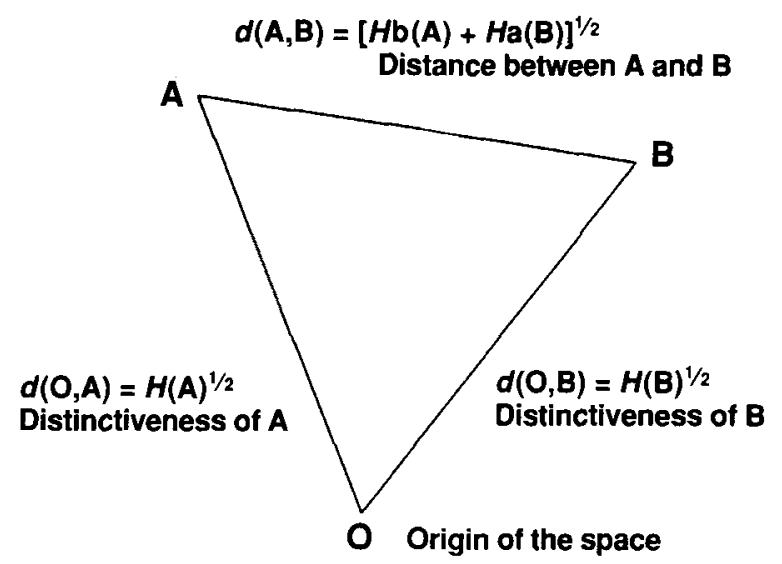

Figure 1. Triangle AOB in euclidean space, illustrating the isomorphism between euclidean distances $d(\mathrm{O}, \mathrm{A}), d(\mathrm{O}, \mathrm{B})$, and $d(A, B)$ and the information measures $H(A) 1^{1 / 2}, H(B)^{1 / 2}$, and $[H \mathbf{a}(\mathrm{B})+\boldsymbol{H b}(\mathrm{A})]^{1 / 2}$. necessarily the same length, $h_{\mathrm{A}} \cos \theta$ may not equal $h_{\mathrm{B}}$ $\cos \theta$, and so the proportion of A represented in $\mathrm{B}$ may not equal the proportion of $B$ represented in $A$.

For the information-distance isomorphism illustrated in Figure 1, the equivalent of $h_{\mathrm{A}} \cos \theta$ is $d(\mathrm{O}, \mathrm{A}) \cos \theta$. (Remember that the angle $\theta$ can be derived from the distance measures in the complete euclidean space.) The information-theory equivalent of $d(\mathrm{O}, \mathrm{A}) \cos \theta$ is $T(\mathrm{~A}: \mathrm{B}) /$ $\sqrt{H(\mathrm{~B})}$, which represents the ratio of information that is common to $A$ and $B[T(A: B)]$ to the square root of the total information in $\mathrm{B}[\sqrt{H(\mathrm{~B})}]$. Its value is zero if $T(\mathrm{~A}: \mathrm{B})$ is zero, and it equals $\sqrt{H(\mathrm{~B})}$ if $T(\mathrm{~A}: \mathrm{B})=H(\mathrm{~B}){ }^{3}$ Therefore, the same structural asymmetry found in the Ekman et al. (1963) space is found in the space generated by information measures based on similarity classification.

There is another structural asymmetry in the information-distance analysis. The interstimulus distance $d(A, B)$ is equivalent to $[\mathrm{Ha}(\mathrm{B})+H \mathrm{~b}(\mathrm{~A})]^{1 / 2}$. This distance is the sum of two asymmetrical components, $\mathrm{Ha}(\mathrm{B}) /[\mathrm{Ha}(\mathrm{B})+$ $H \mathrm{~b}(\mathrm{~A})]^{1 / 2}+H \mathrm{~b}(\mathrm{~A}) /[H \mathrm{a}(\mathrm{B})+H \mathrm{~b}(\mathrm{~A})]^{1 / 2}$. These two terms are different if $H \mathrm{a}(\mathrm{B}) \not \equiv H \mathrm{~b}(\mathrm{~A})$. They can be calculated from the similarity classification data.

Nosofsky $(1985,1992)$ and others have introduced stimulus and response bias terms into symmetrical similarity models in order to accurately predict asymmetric confusions during various performance tasks. The informationdistance measure of distinctiveness, which is the distance from the origin to the stimulus point $[d(0, \mathrm{~A})]$ and which is equivalent to the information measure $\sqrt{H(\mathrm{~A})}$, provides a structural measure of stimulus bias that correlates with directly judged distinctiveness (Donderi, 1988). It has not yet been tested as a component of the prediction for asymmetrical confusions. Thus, there are several ways in which the information-distance isomorphism allows asymmetrical similarity measures to be derived from the distances measured in a similarity space.

Both the Ekman et al. method and the stimulus classification method described here generate complete euclidean spaces for stimulus sets on the basis of observers' responses to the items in the set. The Ekman et al. technique is time-consuming. By comparison, the information-distance isomorphism is economical: The entire stimulus set is partitioned into exclusive classes once by each observer.

\section{THE PROSCALE MDS PROGRAM}

PROSCALE is a new computer program that takes similarity classification data as input, calculates information measures, transforms them into angles and distances, and carries out a factor analysis on the angle cosines that represent the correlations between items in the euclidean factor space.

\section{Principal Components Analysis}

PROSCALE's first step is to average the results from several observers' groupings of the same set of items. ${ }^{4}$ The average item vectors $\mathrm{OA}, \mathrm{OB}, \mathrm{AB}, \ldots$, and their standard deviations, are calculated for observers who 
have classified the same set of items on the basis of similarity. Then, the cosines of the angles between all pairs of the average item vectors are calculated. This matrix of cosines is treated like a matrix of correlations.

A principal components analysis is carried out to determine the eigenvalues and cumulative variances for successive orthogonal dimensions of the space (Gorsuch, 1983). PROSCALE displays these data and then allows the user to choose the number of dimensions to retain for rotation. The retained orthogonal dimensions are then rotated to simple structure using the quartimax criterion, by which a least squares algorithm adjusts the position of the orthogonal dimension coordinates until each item is represented strongly on a small number of dimensions, and each dimension is loaded strongly on a small number of items.

In the conventional factor analysis of a correlation matrix, all item vectors are assumed to have unit length. In the analysis based on similarity grouping, the projection of each item vector onto the rotated coordinates is based on the calculated length of each vector, which corresponds to the distinctiveness of the item.

\section{Output}

PROSCALE's output includes the average item vector lengths $\mathrm{OA}, \mathrm{OB}, \mathrm{AB}$, and so on, and their standard deviations $(S D \mathrm{~s})$. It also includes the interitem distances reconstructed by the Pythagorean formula $\mathrm{AB}=\left[\left(\mathrm{A}_{1}-B_{1}\right)^{2}+\right.$ $\left.\left(\mathrm{A}_{2}-\mathrm{B}_{2}\right)^{2} \ldots+\left(\mathrm{A}_{n}-\mathrm{B}_{n}\right)^{2}\right]^{1 / 2}$ from the projection of each item on the reduced space of rotated principal component dimensions $(1,2, \ldots n)$, where $\left[\mathrm{A}_{1}, \mathrm{~B}_{1}, \mathrm{~A}_{2}, \mathrm{~B}_{2}, \ldots\right.$, $\mathrm{A}_{n}, \mathrm{~B}_{n}$ ] symbolize the projections of Vectors $\mathrm{OA}, \mathrm{OB}$ on the $(1,2, \ldots n)$ retained dimensions of the space. PROSCALE then calculates the percentage of the variance of the original distances accounted for by the reconstructed distances. It also prints the standard error of estimate of the linear regression of the reconstructed distances on the average distances. PROSCALE then prints the coordinates for each item vector in the space of retained and rotated orthogonal dimensions.

PROSCALE also calculates and prints an $S D$ for each item coordinate on each dimension. It calculates the item coordinate $S D$ s from the item vector $S D$. The item vector $S D$ equals the square root of two independent variance components. The first component is the variance of the item vector length averaged across observers. The second component is the square of the standard error of estimate of the linear regression of the reconstructed item vectors on the original item vectors. The item vector $S D$ is itself a vector quantity oriented in the direction of the item vector. This $S D$ is then projected onto each of the rotated orthogonal coordinate dimensions so as to obtain a separate $S D$ component parallel to each coordinate dimension for each item vector.

Unlike some MDS programs, PROSCALE does not generate individual observer weights for sensitivity to each retained dimension (Ramsay, 1991). But it does provide a summary estimate, in the form of a $z$ score, of the variability of each observer's distance data relative to the group average.

\section{Oblique Procrustes Rotation}

PROSCALE allows the user to test sets of variables as targets for oblique dimensions that also span the reduced euclidean space. The variables can be physical or psychological measurements made across the set of items. The number of variables tested must equal the number of retained orthogonal dimensions spanning the space. The "Procrustes" procedure (Gorsuch, 1983) projects a set of oblique vectors into the space and rotates them to a configuration that spans the space and, at the same time, correlates as closely as possible with the set of test variables. PROSCALE extends the Procrustes procedure by also calculating the projection of each of the oblique dimensions onto the rotated orthogonal dimensions of the space.

\section{THEORETICAL AND PRACTICAL COMMENTS}

\section{PROSCALE Results Depend on the Item Set}

PROSCALE's most original feature is either a strength or a weakness, depending on how you look at it. When PROSCALE calculates the uniqueness of a single item and when it calculates the dissimilarity between item pairs, the results depend on the classification of all of the other items in the set and so are not a unique property of the individual items or item pairs. An example should make this clear. One group of observers uses PROSCALE to judge similarity and preference for this mixed set of automobiles: Jaguar XJ6, Mercedes-Benz E300, Dodge Shadow, Plymouth Sundance, Ford Escort, and Chevrolet Cavalier. Another group of observers uses PROSCALE to judge a different set of luxury automobiles: Jaguar XJ6, MercedesBenz E300, Cadillac Seville, BMW 530i, Audi 100CS, and Toyota Lexus (Edmunston, 1994). In the first set, the two luxury cars, Jaguar and Mercedes-Benz, are similar to each other and manifestly dissimilar to the rest of the cars in both style and price. They are a similar pair, and each member of the pair is unique relative to most of the other items in the set. In the second set, Jaguar and Mercedes-Benz are each more like the rest of the luxury cars and so will be judged as less unique, and perhaps even less similar to each other, because of their inclusion in a more homogeneous set of luxurious automobiles.

PROSCALE's uniqueness and dissimilarity measures are explicitly derived from the distribution of similarity groupings over all of the items over all of the observers. It is not a new idea that fundamental measurement depends on the background ensemble or set. In physics, according to Ernst Mach, there is no uniform object motion in empty space, but only motion relative to all of the other masses in the universe (Einstein, 1956, pp. 54-55). In more down-to-earth psychophysics, the results of magnitude estimation scaling and of many other unidimensional psychophysical and psychometric measurements are known to depend quantitatively on the background stim- 
ulus ensemble. Helson (1947) showed that psychophysical judgments were influenced by the range and frequency of exposure of the observer to all of the items in the set to be judged, as well as to the standards used to anchor the response scale (the "adaptation level"). Stevens (1975) recognized that repetition, range, and order biases influenced the results for individual items in magnitude estimation scaling. Poulton (1979) produced a catalogue of biases caused by differences in stimulus range, by differences in response range, and by the number system itself, all of which occur both in category rating scales and in magnitude estimation scales. Berglund, Berglund, and Lindberg (1983) developed a "master scaling" technique in order to eliminate biases across different magnitude estimation scaling sample sets that result from range restriction and other stimulus factors.

Observers participating in both unidimensional and multidimensional scaling procedures experience biases introduced by the particular item set selected for judgment. When MDS observers make a judgment about a pair of items from the set, the other items in the set probably influence the result as an implicit adaptation group. But the grouping task of PROSCALE involves explicit comparison of each item with the entire item set. Since this set may have up to 70 members, the researcher using PROSCALE can select a wide and representative range of items in order to reduce the differential bias introduced by other members of the set.

\section{Multidimensional Classification on a Unidimensional Continuum}

Suppose you have a set of eight pencils. All are sharpened, all are yellow, all are "Eagle," and all are HB. They differ in length: three are short $(3 \mathrm{~cm})$, two are medium $(11 \mathrm{~cm})$, and three are long $(18 \mathrm{~cm})$. Suppose that, following the PROSCALE MDS instructions, observers classify the eight pencils into the above three similarity groups by length. Then, using the information-distance isomorphism previously described, the euclidean distance between the short and long pencils would be 2.05 units, whereas the distance between the medium pencils and either the short or the long pencils would be 2.47 units. This seeming contradiction is resolved when it is seen that the multidimensional distances project onto three orthogonal dimensions of the similarity space. (There are three separate stimulus points in the space: one each for the sets of $3-\mathrm{cm}, 11-\mathrm{cm}$, and $19-\mathrm{cm}$ pencils, plus a separate fourth point for the origin. The distances among four points can be perfectly accommodated in a space of three dimensions.) If the lengths of the pencils are regressed against the coordinates of the three spatial dimensions for the pencils, a multiple regression equation is obtained that describes the projection of the unidimensional measurement scale of pencil length into the three dimensions of the similarity space.

When PROSCALE is used for MDS in a situation that is inherently unidimensional, the projection of the single measured dimension will be embedded in, and can be re- covered from, the higher dimensional similarity space imposed by the sensitivity of PROSCALE to the number of items in each similarity classification. The multidimensional space is generated because PROSCALE measures multidimensional distinctiveness from classifications and then derives multidimensional similarity from multidimensional distinctiveness. The most distinct item in a multidimensional set is the one that is classified with the fewest other items. If there is agreement on the most distinct item across judges, that item will be the least similar item in the similarity space. In the pencils example, there were two medium pencils and three short and three long pencils, so the two medium-length pencils were the most "distinct" and least similar of the pencils.

\section{Magnitude Estimation Scaling}

When a set of items with a single measured property or a single specifiable psychological property (e.g., length, attractiveness) is to be scaled on that property, magnitude estimation scaling (Stevens, 1975) should be used. PROSCALE doubles as a magnitude estimation scaling program. The reference manual provides alternative instructions, and the program provides alternative algorithms for generating magnitude estimation scales. In this role, PROSCALE retains the advantage of handling data from as many as 50 observers and as many as 70 items. PROSCALE calculates the geometric mean and the $95 \%$ confidence limits of the magnitude estimation ratings for each item. In addition, the values of as many as 20 independent variables can be associated with each item. PROSCALE includes a multiple regression routine that displays and saves the regression coefficients and related statistics for any combination of the 20 independent variables regressed against both the geometric mean of the magnitude estimation rating and the $\log$ of the geometric mean rating.

PROSCALE's magnitude estimation scaling routine should be used to measure a set of items on a single psychological property, and the MDS algorithm should be used to scale sets of items with multiple, and possibly unknown, psychological properties.

\section{Applications}

Even before the PROSCALE program became available, similarity classification studies of 45,49 , and 70 items had been carried out on samples from the pulp and paper industry (e.g., Donderi \& Aspler, 1994). There are many other large data sets whose psychological similarity structure is potentially interesting (e.g., countries, politicians, products, buildings, language samples, archaeological artifacts, and faces). MDS with PROSCALE may be the technique of choice for analyzing large sample sets like these, especially when data have been collected from many observers.

\section{Documents, Languages, and Platforms}

PROSCALE is described for users in two publications (Donderi, 1995a, 1995b). The Proscale Reference Man- 
ual provides background theoretical information. How to Collect Data for PROSCALE explains in detail how to set up a study, how to collect data, and how to prepare them for analysis. PROSCALE instructions and procedures were written and its interface was designed so that data collection, data entry, and basic analyses can be carried out by laboratory technicians.

PROSCALE information analysis routines are written in FORTRAN. The program uses IMSL factor analysis subroutines to carry out the principal components analysis, the factor rotation, and the Procrustes rotation of oblique coordinates to match arbitrary variables. The program uses Interacter routines to handle input-output displays. The program requires an 80306 or higher computer equipped with a math coprocessor. It runs under DOS or in a DOS window under Windows.

\section{Availability}

FORTRAN source code and instructions for PROSCALE are available free from the author. The source code was compiled with the Lahey EM-32 compiler. The code calls IMSL statistical subroutines that must be available for linkage if the source code is to execute. A diskette containing a complete executable version of PROSCALE as well as the source code, sample data, and instructions is also available.

\section{REFERENCES}

AtTnEave, F. (1959). Applications of information theory to psychology. New York: Holt, Rinehart \& Winston.

Berglund, B., Berglund, O., \& LindberG, S. (1983). Master scaling of environmental loudness (Rep. No. 610). Stockholm: University of Stockholm, Department of Psychology.

Cleveland, W. W., Harris, C. S., \& McGill, R. (1983). Experiments on quantitative judgments of graphs and maps. Bell System Technical Journal, 62, 1659-1674.

DONDERI, D. C. (1988). Information measurement of distinctiveness and similarity. Perception \& Psychophysics, 44, 576-584.

Donderi, D. C. (1995a). How to collect data for PROSCALE. Pointe Claire, PQ: Canada: Pulp and Paper Research Institute of Canada.

Donderi, D. C. (1995b). PROSCALE 3.0 reference manual. Pointe Claire, PQ: Canada: Pulp and Paper Research Institute of Canada.

Donderi, D. C., \& Aspler, J. A. (1994). Correlates of preference and similarity in 45 printed samples from nine newsprint machines. Nordic Pulp \& Paper Journal, 2, 88-93.

Edmunston, P. (1994). Lemon-aid new car guide, 1994. Toronto: Stoddart

EINSTEIN, A. (1956). The meaning of relativity (6th ed.). London: Methuen.
Ekman, G., Engen, T., Künnapas, T., \& Lindman, R. (1963). A quantitative principle of qualitative similarity (Rep. No. 152). Stockholm: University of Stockholm, Psychological Laboratory.

GARNER, W. R. (1974). The processing of information and structure. Potomac, MD: Erlbaum.

Gorsuch, R. L. (1983). Factor analysis. Hillsdale, NJ: Erlbaum.

Helson, H. (1947). Adaptation-level as a frame of reference for prediction of psychophysical data. American Journal of Psychology, 60, 1-29.

Mellers, B. A., \& Cooke, A. D. J. (1996). The role of task and context in preference measurement. Psychological Science, 7, 76-82.

NosOFsKY, R. [M.] (1985). Overall similarity and the identification of separable-dimension stimuli: A choice model analysis. Perception \& Psychophysics, 38, 415-432.

NosofSky, R. M. (1991). Stimulus bias, asymmetric similarity, and classification. Cognitive Psychology, 23, 94-140.

Nosofsky, R. M. (1992). Similarity scaling and cognitive process models. Annual Review of Psychology, 43, 25-53.

PARKE, N. G., \& SAMPSON, E. W. (1951). Distance and equivalence in sequence space (Lab. Note E4080). Cambridge, MA: Air Force Cambridge Research Laboratories.

Poulton, E. C. (1979). Models for biases in judging sensory magnitude. Psychological Bulletin, 86, 777-803.

RAMSAY, J. O. (1991). Multiscale manual: Extended version. McGill University.

ShEPARD, R. N. (1964). Attention and the metric structure of the stimulus space. Journal of Mathematical Psychology, 1, 54-57.

Spence, I., \& Domoney, D. W. (1974). Single subject incomplete designs for nonmetric multidimensional scaling. Psychometrika, 39, 469-490.

StEvens, S. S. (1975). Psychophysics: Introduction to its perceptual, neural and social prospects. New York: Wiley.

TVERSKy. A, (1977). Features of similarity. Psychological Review, 84, 327-352.

TVERSKY, A., \& GAti, I. (1982). Similarity, separability, and the triangle inequality. Psychological Review, 89, 123-154.

\section{NOTES}

1. In conventional MDS, the origin is simply treated as an extra item.

2. See Attneave (1959), which is a standard reference source for the information measurement terms defined here.

3. The measure of information transmitted between $\mathrm{A}$ and $\mathrm{B}, T(\mathrm{~A}: \mathrm{B})$, is defined in information theory terms in Attneave (1959) and is derived from stimulus classifications in Donderi (1988).

4. Donderi (1988) had observers classify the items five times, "a different way each time, using the clearest way the first time, down through to the least important way the fifth time." Results obtained using only the first classification per observer turned out to correlate more highly with conventional MDS results than did the average of five classifications per observer; therefore, subsequent applications have required only a single classification from each observer.

(Manuscript received December 11, 1995; revision accepted for publication December $20,1996$. 